\title{
Harms associated with single unit perioperative transfusion: retrospective population based analysis
}

\author{
Elizabeth L Whitlock, ${ }^{1}$ Helen Kim, ${ }^{1}$ Andrew D Auerbach²
}

'Department of Anesthesia and Perioperative Care, University of California, CA 94143-0648, USA 2Division of Hospital Medicine, Department of Medicine, University of California, San Francisco, CA 94143, USA

Correspondence to: E Whitlock elizabeth.whitlock@ucsf.edu

Additional material is published online only. To view please visit the journal online (http://dx.doi. org/10.1136/bmj.h3037)

Cite this as: BMJ 2015;350:h3037 doi: 10.1136/bmj.h3037

Accepted: 23 May 2015

\section{ABSTRACT}

OBJECTIVE

To determine whether perioperative transfusion of as little as one unit of packed red blood cells in the operating room or the day after surgery is associated with measurably increased odds for perioperative ischemic stroke and myocardial infarction.

\section{DESIGN}

Retrospective cohort study of hospital administrative data.

\section{SETTING}

346 hospitals in the United States participating in the claims based Premier Perspective database from 1 January 2009 to 31 March 2012.

\section{PARTICIPANTS}

1583819 adults who underwent non-cardiac, nonintracranial, non-vascular surgery and required a stay of at least one night in hospital and did not receive packed red blood cells on days two to seven after surgery.

\section{INTERVENTION}

Transfusion of packed red blood cells on the day of surgery or one day after by exposure categories (none or one, two, three or four or more units).

\section{MAIN OUTCOME MEASURES}

The composite outcome of stroke/myocardial infarction was defined as ischemic stroke, ST elevation myocardial infarction, ventricular tachycardia, or ventricular fibrillation during index admission or as a primary diagnosis for readmission within 30 days. Ventricular tachycardia/ventricular fibrillation were included as a surrogate for myocardial infarction.

RESULTS

41421 (2.6\%) patients received at least one unit of packed red blood cells within 48 hours of surgery, and $8044(0.51 \%)$ experienced the composite outcome of stroke/myocardial infarction. Patients who were transfused were older, more likely to be women, and had more comorbid disease. Hierarchical logistic regression adjusted for comorbidities and demographics with random effects by hospital showed

\section{WHAT IS ALREADY KNOWN ON THIS TOPIC}

Stored blood undergoes biochemical and morphological changes that could impair post-transfusion delivery of oxygen and contribute to ischemic outcomes

Perioperative hemorrhage is associated with ischemic stroke and myocardial infarction, but the potential contribution of transfusion is unknown

\section{WHAT THIS STUDY ADDS}

There is an association between perioperative transfusion of as little as one unit of blood and ischemic stroke or myocardial infarction that transfusion of as little as one unit was associated with an odds ratio of 2.33 (95\% confidence interval 1.90 to 2.86 ) for perioperative stroke/myocardial infarction, and the odds of stroke/myocardial infarction markedly increased with transfusion of four or more units. Subgroup analysis limiting the cohort to one of several common surgical procedures, excluding those who received two or more units, or excluding who received transfusion on postoperative day one showed substantially similar results, as did a matched propensity score analysis. Two methods of modeling unmeasured confounders suggest an odds ratio of $>10$ with imbalance of up to $47 \%$ between patients who did and did not receive transfusion would be required to invalidate our results.

\section{CONCLUSIONS}

A perioperative transfusion of one unit of packed red blood cells is associated with increased odds of perioperative ischemic stroke and/or myocardial infarction, even after adjustment for a wide range of factors in our data and despite extensive sensitivity analyses.

\section{Introduction}

Nearly 14 million units of whole blood or packed red blood cells were transfused in the United States in 2011, the most recent year for which data are available. ${ }^{1}$ Recent work showed that transfusion of four or more units of packed red blood cells was associated with 2.5-fold increased odds of perioperative stroke or myocardial infarction. ${ }^{2}$ Perioperative stroke and myocardial infarction, while rare, increase the risk of perioperative mortality by threefold to fourfold, and have far-reaching implications for postoperative function, quality of life, and hospital costs. ${ }^{3-5}$ It is not known whether smaller volume transfusions-such as one to three units-are similarly associated with an increased risk for these outcomes, although there is a far greater population exposed to perioperative transfusion in the absence of hemorrhage per se.

Stored packed red blood cells become increasingly inflexible and more adherent to vascular endothelium with time, ${ }^{6}$ interfere with hypoxic vasodilation, ${ }^{7}$ and increase platelet reactivity and aggregation. ${ }^{8}$ Within the first 24 hours, transfusion with packed red blood cells typically fails to improve tissue oxygenation and might in fact decrease it. ${ }^{9-11}$ These data provide a theoretical association between perioperative transfusion and ischemic events, but large scale clinical data unconfounded by the potential contribution of perioperative hemorrhage are lacking.

To test the hypothesis that there is a measurably increased risk of perioperative ischemic stroke or 
myocardial infarction associated with transfusion of as little as one unit of packed red blood cells, we conducted a retrospective cohort study using a large commercially available dataset.

\section{Methods}

Data source

The Premier Perspective database (Premier, Charlotte, $\mathrm{NC}$ ) is an anonymized, fee supported, voluntary dataset created to support investigation of healthcare quality and utilization. It captures about $20 \%$ of all hospital discharges from acute care in the US. Data collected include standard hospital discharge file data and a date stamped log of all billed items, including drugs, laboratory and other diagnostic tests, and therapeutic services provided during the admission. The data are collected electronically and regularly audited by Premier. Procedure and comorbidity data are provided as ICD-9 (international classification of diseases, ninth revision) codes. Procedure codes are stratified into primary and secondary; discharge diagnosis codes are designated as primary or secondary and might have a modifier for "present on admission." Our dataset included up to 25 diagnosis codes for each patient. A median of five (interquartile range three to eight) diagnoses were present on admission.

\section{Patients}

Patients in our analysis were admitted between 1 January 2009 and 31 March 2012, were aged $\geq 18$, and were admitted for at least one night. We considered only the first episode of care for patients who had more than one admission during the study period. To attempt to exclude emergency cases, we did not include patients admitted from the emergency department or from hospital transfer. We also did not include patients with a primary diagnosis of intracranial injury or those undergoing obstetrical, cardiac, intracranial (including peritoneal shunt revision), or major vascular procedures, as those patients might have specific risks for stroke or myocardial infarction related to the procedure or diagnosis. We then excluded patients with a primary procedure code for a non-surgical procedure (such as intubation, thoracentesis, lumbar puncture) and patients with unknown sex. To avoid confounding by transfusions later in the admission, we also excluded patients who underwent transfusion on days two to seven after surgery.

\section{Patient involvement}

There was no patient involvement in this study.

\section{Variables}

Hospital level data directly collected by Premier include number of beds, urban or rural location, and teaching hospital status. Patient related values include year and month of admission, patient age, race/ethnicity, sex, length of stay and total hospital costs, insurance payor (whether the patient has health insurance provided through a private company, through the US government programs Medicare or Medicaid, or is uninsured), 30 day readmission, mortality (during index admission or on readmission), primary and secondary discharge diagnoses and primary procedure, and admission diagnosis if readmitted within 30 days. Diagnoses coded as present on admission were further categorized according to the methods of Elixhauser for obtaining pre-existing comorbid conditions from ICD-9 data. ${ }^{12}$

Details on transfusion of red blood cells were derived from billing data. Because some units were documented as partially transfused, the number of units of packed red blood cells was rounded up to the nearest integer, reflecting the number of units to which the patient was exposed. We included autologous, directed donor, and whole blood units, which in composite represented $3.3 \%$ of all units transfused.

\section{Outcome definition}

The primary outcome for this study was a composite of ischemic stroke, myocardial infarction, and ventricular tachycardia or fibrillation, which was included as a potential surrogate for coronary ischemia with or without myocardial infarction. ${ }^{13}$ Patients with a discharge diagnosis of stroke (ICD-9 433.x1, 434.x1, 977.02), initial episode of care for myocardial infarction (ICD-9 410.x1), or ventricular tachycardia/ventricular fibrillation (ICD-9 427.1 or $427.4 x$ ), or any of these diagnoses as a primary diagnosis for a subsequent readmission within 30 days, were considered to have had the primary outcome of interest. Strokes, myocardial infarctions, or episodes of ventricular tachycardia/ventricular fibrillation coded as present on admission during the initial admission were not included in the primary outcome. These ICD-9 codes, and therefore the outcome definition, do not include hemorrhagic stroke, subarachnoid hemorrhage, unspecified or subsequent episode of care for myocardial infarction, or atrial fibrillation.

\section{Statistical analysis}

Data management was conducted in SAS version 9.2 (SAS Institute, Cary, NC), and statistical analysis in Stata 13.1 (StatCorp LP, College Station, TX). Where not specified, $\mathrm{P}<0.05$ was considered to indicate significance.

\section{Primary logistic regression model}

Variables were considered for entry into the model on the basis of face validity and a literature search to identify factors previously shown to be associated with perioperative stroke and myocardial infarction. A manual forward iterative technique was used to develop the multivariable logistic regression model, with the likelihood ratio test and area under the receiver operator characteristic (ROC) curve used to evaluate significant improvement of the model. We included in the model variables that achieved significant improvement in likelihood ratio at $\mathrm{P} \leq 0.025$ and improved the area under the ROC curve by 0.005 .

An initial non-hierarchical logistic regression model was first created based on stratification of the 
data into a population without cardiovascular risk factors (defined a priori as prior stroke or transient ischemic attack, coronary artery disease, congestive heart failure, atrial fibrillation, diabetes with chronic complications, renal failure, cardiac valvular disease, peripheral vascular disease, or chronic anticoagulant use). Significant associations of risk factors in the lower risk population were assumed to extend to the higher risk patients, and cardiovascular and other comorbidities were added into the model and selected for according to the above criteria. We generated a composite variable, incorporating history of congestive heart failure, atrial fibrillation, diabetes with chronic complications, and/or renal failure, because these comorbidities individually had low population prevalence $(<5 \%)$ and grouping them as a composite variable compared with including them individually had minimal impact on area under the ROC curve, but in aggregate those factors produced an improvement consistent with inclusion according to the above criteria.

We then investigated potential interactions by sequential examination of population strata based on important model components (comorbidities or demographic factors). Interactions among the variables were investigated by using interaction terms in the logistic regression analysis and the margins and marginsplot commands in Stata, and significant interactions were retained.

Finally, the logistic regression model thus created was further refined by adjustment for random effects within hospitals with Stata's meqrlogit command, which uses the $\mathrm{QR}$ decomposition of the variance components matrix to improve convergence. As the hierarchical component changed the odds ratios for the primary predictor by $>10 \%$, it was retained. The final hierarchical regression model had an area under the ROC curve of 0.833 and no evidence of collinearity with a maximum variance inflation factor of 1.37 .

\section{Subgroup analyses}

The hierarchical model was also used to investigate the association between transfusion and stroke/myocardial infarction in surgical subgroups-total or partial colectomy, small bowel resection, hip or knee replacement or revision, laminectomy and/or fusion of the spine, and hysterectomy-on the basis of ICD-9 codes.

We used the same model in a subgroup restricted to patients who received no more than one unit of blood and in a subgroup in which we additionally excluded any patient who received transfusion on the day after surgery (that is, we included only patients who received transfusions on the day of surgery and those who had never received transfusion; the primary analysis already excluded patients who received transfusion on days two to seven after surgery). Finally, we analyzed outcomes individually (that is, stroke, myocardial infarction not including ventricular tachycardia/ ventricular fibrillation, and ventricular tachycardia/ ventricular fibrillation) to ensure odds ratios for all three outcomes were consistent and in the same direction, to confirm the appropriateness of the composite outcome analysis.

\section{Propensity score analysis}

We performed a propensity score analysis to complement the hierarchical logistic regression primary analysis. We used fixed logistic regression with clustering by hospital to compute a propensity score for any blood transfusion on the day of surgery or one day after based on all patient level factors in the primary model as well as chronic anticoagulant use, chronic lung disease, peripheral vascular disease, cancer, and inflammatory arthritis, again with random effects by facility. These factors were determined a priori on the basis of face validity and significant $(\mathrm{P}<0.025)$ univariate differences between groups who did and not receive transfusion. To improve predictive power of the propensity model, we additionally assembled a composite maximum surgical blood ordering schedule (MSBOS) using data from a published ICD-9-indexed partial MSBOS $^{14}$ and supplemented with data from the MSBOS used at the University of California-San Francisco (UCSF). ${ }^{15}$ This schedule is indexed by a proprietary code used internally by our electronic medical record, which could not be linked to ICD-9 codes; accordingly, procedures with a frequency of $>1000$ in the dataset were manually matched to UCSF MSBOS values. A total of 157592 patients undergoing 1512 distinct procedures were not matched to a MSBOS value. Area under the ROC curve for the propensity score model was 0.715 . Then, 1 -to-1 greedy matching without replacement was used to match all 41421 patients who received a transfusion to 41421 patients who did not, within a propensity score caliper distance of 0.01 or less in all cases. In the case of a tie, one patient was randomly selected from among the group of patients who did not receive a transfusion with well matched propensity scores.

We used this propensity score matched cohort in two ways. First, we performed univariate analysis comparing stroke/myocardial infarction rate between the transfused and non-transfused matched population. Then, because risk factors for stroke/myocardial infarction were non-significantly over-represented in the transfused cohort (leading to a possible overall bias toward stroke/myocardial infarction in the transfused group), we entered the matched cohort into the hierarchical logistic regression model developed above to adjust for the imbalanced covariates. Two propensity score sensitivity analyses are reported in table B in the appendix: one eliminating patients without a matched blood ordering schedule value (that is, inclusion only of patients with a known value), and one that does not use the blood ordering schedule. Results of those models were not substantially different to the model reported here.

\section{Population attributable risk}

The population attributable fraction (PAF) is a measurement of incremental increase in outcome rate 
Participant flow in study of perioperative transfusion and outcomes
Overnight admissions with at least on primary procedure when primary diagnosis was not cardiac or intracerebral $(n=2827068)$

\section{Exclusions ( $n=1243$ 249):}

By type of procedure $(n=1187900)$ :

Craniotomy, VP shunt revision $(n=1606)$

Cardiac $(n=9340)$

Major vascular $(n=80576)$

Obstetrical ( $\mathrm{n}=992$ 923)

Minor procedures, including tracheostomy $(n=103455)$

Sex unknown $(n=164)$

Transfusion other than on day of surgery or day after $(\mathrm{n}=55$ 185):

Postoperative day $\geq 2(n=49776)$

Postoperative day $\geq 5(n=5409)$

Final population $(n=1583819)$

Stroke or myocardial infarction $(n=8044)$

Transfusion on day of surgery or day after $(n=41421)$

Table 1 | Characteristics of 1583819 patients undergoing surgery between January 2009 and March 2012 by packed red blood cell transfusion status. ${ }^{*}$ Figures are numbers (percentage) unless stated otherwise

\begin{tabular}{|c|c|c|}
\hline Variable & Not transfused & Transfused \\
\hline No of patients & 1542398 & 41421 \\
\hline Mean (SD) age (years) & $58.8(16.0)$ & $65.4(15.4)$ \\
\hline Men & $594328(38.5)$ & $12833(31.0)$ \\
\hline \multicolumn{3}{|l|}{ Race/ethnicity: } \\
\hline Black & $149147(9.7)$ & $4278(10.3)$ \\
\hline Hispanic & $38665(2.5)$ & $905(2.2)$ \\
\hline Other & 236701 (15.4) & 6179 (14.9) \\
\hline White & $1117885(72.5)$ & $30059(72.6)$ \\
\hline \multicolumn{3}{|l|}{ Comorbidities: } \\
\hline Cerebrovascular disease & $11018(0.71)$ & $535(1.29)$ \\
\hline CAD or MI history & $177604(11.5)$ & 7017 (16.9) \\
\hline Obesity & $231443(15.0)$ & $5081(12.3)$ \\
\hline Smoking & $193717(12.6)$ & $3749(9.1)$ \\
\hline Pre-existing anemia & $200003(13.0)$ & $12266(29.6)$ \\
\hline Cardiovascular risk factors $\dagger$ & $287766(18.7)$ & $11450(27.6)$ \\
\hline Atrial fibrillation & $73203(4.8)$ & $3322(8.0)$ \\
\hline CHF & $41060(2.7)$ & $2258(5.5)$ \\
\hline Diabetes with chronic complications & $27393(1.8)$ & $1153(2.8)$ \\
\hline Renal failure & $66260(4.3)$ & $3726(9.0)$ \\
\hline \multicolumn{3}{|l|}{ Surgery type: } \\
\hline Spine/PNS & 201417 (13.1) & $4052(9.8)$ \\
\hline General & $385284(25.0)$ & 8135 (19.6) \\
\hline Thoracic & $15440(1.0)$ & $751(1.8)$ \\
\hline Urogenital & $93960(6.1)$ & $2704(6.5)$ \\
\hline Gynecological & $199501(12.9)$ & $3829(9.2)$ \\
\hline Orthopedic & $599600(38.9)$ & $20849(2.7)$ \\
\hline Integumentary & $47196(3.1)$ & $1101(2.7)$ \\
\hline Readmitted within 30 days & $113814(7.4)$ & $5412(13.1)$ \\
\hline \multicolumn{3}{|l|}{ Outcomes: } \\
\hline Stroke & $2726(0.18)$ & $153(0.37)$ \\
\hline MI or VT/VF & $4914(0.32)$ & $348(0.84)$ \\
\hline Stroke, MI, or VT/VF & $7548(0.49)$ & $496(1.20)$ \\
\hline Death & $10448(0.68)$ & $1220(3.0)$ \\
\hline \multicolumn{3}{|c|}{$\begin{array}{l}\text { Transfusion amount (units) (percentage of all } \\
\text { patients who underwent transfusion): }\end{array}$} \\
\hline 1 & - & $12847(31.0)$ \\
\hline 2 & - & $21642(52.3)$ \\
\hline 3 & - & $2926(7.1)$ \\
\hline$\geq 4$ & - & $4006(9.7)$ \\
\hline Median (IQR) hospital costs (\$) & $12255(7540-18167)$ & $18383(12825-27904)$ \\
\hline
\end{tabular}

$\mathrm{CAD}=$ coronary artery disease $\mathrm{MI}=$ myocardial infarction: $\mathrm{CHF}=$ congestive heart failure. $\mathrm{PNS}=$ peripheral nervous system; VT/VF=ventricular tachycardia or ventricular fibrillation.

*All P values for comparison between transfused and non-transfused patients were $<0.001$.

tIncludes patients who have at least 1 of atrial fibrillation, CHF, diabetes with chronic complications, or renal failure. experienced by patients with a certain risk factor compared with those without that risk factor. The adjusted fraction was calculated according to the methods of Greenland and Drescher ${ }^{16}$ with the Stata command punaf, which performs post-estimation calculations on models that produce conditional arithmetic means. The command is not compatible with interaction terms or hierarchical models, so we ran punaf on a simplified regression model omitting interaction terms and with clustering (not random effects) by hospital.

Estimating the impact of an unmeasured confounder We used two methods to model the impact of a possible unmeasured covariate unequally distributed between the patients who did and did not undergo transfusion and the required strength of association with stroke/myocardial infarction required to invalidate the association between transfusion and this outcome. The first was according to methods described by Lin and colleagues, ${ }^{17}$ who use the algebraic relation between the modeled effect size in the primary model and the effect size and distribution of a hypothetical unmeasured confounder to model its impact. The second technique followed the methods of Sturmer and colleagues, which leverages propensity score calibration methods. ${ }^{18}$

\section{Results}

\section{Study population}

A total of 1583819 patients from 346 hospitals underwent major surgery during the study dates, of whom $41421(2.6 \%)$ received packed red blood cells on the day of surgery or the day after but not in the next six days (that is, postoperative days 2-7) (figure). Patients who received a transfusion were older, more likely to be women, had more medical comorbidities and higher hospital costs, and were more likely to experience ischemic stroke, myocardial infarction, and 30 day readmission or death (table 1 ). The most common transfusion volume was two units $(52.3 \%$ of all patients who received transfusion). Within 30 days of surgery, 8044 patients (0.51\%) experienced stroke or myocardial infarction; 5165 had a myocardial infarction, 2782 had a stroke, and 97 had both. Table 2 shows the characteristics of the hospitals attended.

\section{Primary hierarchical logistic regression model for stroke/myocardial infarction}

After adjustment for other patient comorbidity and demographic factors that could affect the risk of ischemic stroke or myocardial infarction, transfusion of packed red blood cells was associated with increasing odds of perioperative stroke/myocardial infarction: the odds were about twofold for one or two units, threefold for three units, and fivefold for four units (table 3). History of cerebrovascular disease (stroke or transient ischemic attack) was associated with the largest adjusted odds ratio for the composite outcome (8.20, 95\% confidence interval 7.58 to 8.87 ). 


\begin{tabular}{|c|c|c|}
\hline Variable & Not transfused & Transfused \\
\hline No of patients & 1542398 & 41421 \\
\hline \multicolumn{3}{|l|}{ Insurance payor: } \\
\hline Uninsured & 53968 (3.5) & $1059(2.6)$ \\
\hline Indemnity & $196747(12.8)$ & $4122(10.0)$ \\
\hline Managed care, capitated & $12007(0.78)$ & $267(0.64)$ \\
\hline Managed care, non-capitated & $507466(32.9)$ & $9397(22.7)$ \\
\hline Medicaid & $100180(6.5)$ & $2194(5.3)$ \\
\hline Medicare & $647847(42.0)$ & $23975(57.9)$ \\
\hline Other & $24183(1.6)$ & $407(0.98)$ \\
\hline Urban setting & $1383335(89.7)$ & $36943(88.9)$ \\
\hline Teaching hospital & $604288(39.2)$ & $15882(38.3)$ \\
\hline \multicolumn{3}{|l|}{ Hospital size (No of beds): } \\
\hline $0-99$ & $46959(3.0)$ & $894(2.2)$ \\
\hline $100-199$ & $173322(11.2)$ & $4717(11.4)$ \\
\hline $200-299$ & 289209 (18.8) & $7360(17.8)$ \\
\hline 300-399 & $328792(21.3)$ & $10239(24.7)$ \\
\hline 400-499 & $270400(17.5)$ & $5074(12.3)$ \\
\hline$>500$ & $433716(28.1)$ & $13137(31.7)$ \\
\hline \multicolumn{3}{|l|}{ Provider region: } \\
\hline Midwest & $268968(17.4)$ & $3176(7.7)$ \\
\hline Northeast & 279331 (18.1) & $10576(25.5)$ \\
\hline South & $648436(42.0)$ & $18656(45.0)$ \\
\hline West & $345663(22.4)$ & $9013(21.8)$ \\
\hline
\end{tabular}

\section{Subgroup and sensitivity analyses}

Subgroup analyses limiting the model to five common types of surgery (table 4) yielded wider confidence intervals for most predictors but did not substantially affect the point estimates for the impact of transfusion on stroke/myocardial infarction. Other strong predictors (cardiovascular risk factors, cerebrovascular disease, and coronary artery disease) remained important in the model; interaction terms were not significant (data not shown).

Sensitivity analysis excluding patients transfused two or more units of blood showed an odds ratio for 1 unit transfusion of 2.03 (95\% confidence interval 1.55 to 2.66); other covariates were not substantially different compared with the results for the whole dataset model (table A in appendix). A sensitivity analysis with only transfusions on the day of surgery as the primary predictor and excluding patients transfused on day one after surgery also showed substantially similar results, with wider confidence intervals (table A in appendix). Finally, application of the hierarchical logistic regression model to individual outcomes (that is, ischemic stroke, myocardial infarction excluding ventricular tachycardia/ventricular fibrillation, and ventricular tachycardia/ventricular fibrillation) also yielded substantially similar results for the association with transfusion, although the strength of association between perioperative myocardial infarction or ventricular tachycardia/ventricular fibrillation and history of cerebrovascular disease, and the strength of association between perioperative stroke and history of coronary artery disease, were weaker than in the composite outcome model (table A in appendix).

\section{Propensity score analysis}

A propensity score matched analysis confirmed the increased risk of stroke/myocardial infarction in patients who received packed red blood cells compared with those with similar probability of transfusion who did not receive blood (table 5; table B in appendix). Odds ratios for stroke/myocardial infarction in the propensity score cohort from both the unadjusted and adjusted analyses were substantially similar to those derived from the primary hierarchical logistic regression analysis. Given the absolute risk difference of $0.37 \%$ in the matched cohort, and if we assume a causal relation, unnecessary single unit transfusion of 267 patients would be expected to result in one additional stroke/myocardial infarction event.

\section{Population attributable fraction}

The adjusted population attributable fraction for packed red blood cell transfusion was 2.4\% (95\% confidence interval $1.8 \%$ to $3.0 \%$ ). In comparison, it was $9.7 \%(8.6 \%$ to $10.7 \%$ ) for cerebrovascular disease, $20.2 \%$ (18.5\% to $22.0 \%$ ) for coronary artery disease history, and $43.3 \%$ (41.1\% to $45.4 \%$ ) for all comorbidity covariates retained in the regression model (that is, cerebrovascular disease, coronary artery disease, cardiovascular risk factors, obesity, anemia and smoking).

\section{Estimating the impact of an unmeasured confounder} Two methods of modeling an unmeasured confounder showed that, to account for the $133 \%$ or greater increase in adjusted odds of stroke or myocardial infarction, an unmeasured confounder with an odds ratio of $>10$ would need to be present in $47 \%$ more patients who underwent transfusion than in patients who did not receive a transfusion (for example, $10.0 \%$ in patients not undergoing transfusion $v 14.7 \%$ in those undergoing transfusion) to remove the observed associations between packed red blood cell transfusion and worse outcomes.

\section{Discussion}

In this observational study of almost 1.6 million patients, perioperative transfusion of a single unit of packed red cells was significantly associated with perioperative ischemic stroke, myocardial infarction, or ventricular tachycardia/ventricular fibrillation (as a surrogate for coronary ischemia). The association we observed (adjusted odds ratio 2.33) was substantial, and similar to the odds ratio for coronary artery disease or one or more of several risk factors for stroke/myocardial infarction (congestive heart failure, atrial fibrillation, diabetes with chronic complications, or renal failure) in this dataset; the fraction of risk attributable to transfusion itself, however, was substantially smaller than for non-modifiable comorbid conditions. 


\begin{tabular}{|c|c|c|c|}
\hline Variable & $\begin{array}{l}\text { No (\%) without stroke/ } \\
\text { MI }(n=1575775)\end{array}$ & $\begin{array}{l}\text { No (\%) with stroke/ } \\
\text { MI }(n=8044)\end{array}$ & Multivariate $O R$ \\
\hline 0 & $1524850(97.4)$ & $7548(93.8)$ & (reference) \\
\hline 1 & $12715(0.81)$ & $132(1.6)$ & $2.33(1.90$ to 2.86$)$ \\
\hline 2 & $21420(1.4)$ & $222(2.8)$ & 2.37 (2.00 to 2.81$)$ \\
\hline 3 & $2881(0.18)$ & $45(0.56)$ & $3.13(2.28$ to 4.31$)$ \\
\hline$\geq 4$ & $3909(0.25)$ & $97(1.2)$ & 4.87 (3.86 to 6.14$)$ \\
\hline Mean (SD) age & $58.9(16.0)$ & $71.2(12.8)$ & $1.43(1.40$ to 1.46$) \dagger$ \\
\hline Men (reference: women) & $602781(38.3)$ & $4830(54.5)$ & $1.46(1.40$ to 1.53$)$ \\
\hline \multicolumn{4}{|l|}{ Race/ethnicity: } \\
\hline Black & $152529(9.7)$ & $896(11.1)$ & 1.45 (1.34 to 1.56$)$ \\
\hline Hispanic & $39460(2.5)$ & $110(1.4)$ & $0.77(0.63$ to 0.94$)$ \\
\hline Other & $241783(15.3)$ & $1097(13.6)$ & 0.99 (0.92 to 1.08$)$ \\
\hline White & $1142003(72.5)$ & $5941(73.9)$ & (reference) \\
\hline \multicolumn{4}{|l|}{ Insurance payor: } \\
\hline Uninsured & $54847(3.5)$ & $180(2.2)$ & $1.62(1.38$ to 1.91$)$ \\
\hline Indemnity & $200381(12.7)$ & $488(6.1)$ & $1.12(1.00$ to 1.25$)$ \\
\hline Managed care, capitated & $12245(0.78)$ & $29(0.36)$ & 0.97 (0.66 to 1.42$)$ \\
\hline Managed care, non-capitated & $515841(32.7)$ & $1022(12.7)$ & (reference) \\
\hline Medicaid & $102009(6.5)$ & $365(4.5)$ & $1.72(1.52$ to 1.95$)$ \\
\hline Medicare & $665941(42.3)$ & $5881(73.1)$ & $1.36(1.26$ to 1.47$)$ \\
\hline Other & $24511(1.6)$ & $79(0.98)$ & $1.42(1.11$ to 1.80$)$ \\
\hline \multicolumn{4}{|l|}{ Comorbidities: } \\
\hline Cardiovascular risk factors $\ddagger$ & $295042(18.7)$ & 4174 (51.9) & 2.43 (2.30 to 2.56$)$ \\
\hline Cerebrovascular disease & $10641(0.68)$ & $912(11.3)$ & 8.20 (7.58 to 8.87$)$ \\
\hline CAD or MI history & $181386(11.5)$ & $3235(40.2)$ & 2.05 (1.95 to 2.16$)$ \\
\hline Obesity & 235634 (15.0) & $890(11.1)$ & 0.86 (0.80 to 0.93$)$ \\
\hline Smoking & $196536(12.5)$ & $940(11.7)$ & $1.26(1.17$ to 1.35$)$ \\
\hline Anemia & $210558(13.4)$ & $1711(21.3)$ & $1.07(1.01$ to 1.14$)$ \\
\hline \multicolumn{4}{|l|}{ Surgery type: } \\
\hline Spine/PNS & $204743(13.0)$ & $726(9.0)$ & $1.15(1.06$ to 1.25$)$ \\
\hline General & $389943(24.8)$ & $3476(43.2)$ & 2.54 (2.41 to 2.68$)$ \\
\hline Thoracic & $15882(1.0)$ & $309(3.8)$ & 3.68 (3.25 to 4.16$)$ \\
\hline Urogenital & $96129(6.1)$ & $535(6.7)$ & $1.15(1.05$ to 1.27$)$ \\
\hline Gynecologic & $203123(12.9)$ & 207 (2.6) & $0.78(0.67$ to 0.90$)$ \\
\hline Orthopedic & $617863(39.2)$ & $2586(32.2)$ & (reference) \\
\hline Integumentary & $48092(3.1)$ & $205(2.6)$ & $1.27(1.10$ to 1.47$)$ \\
\hline \multicolumn{4}{|c|}{ Interaction terms (any transfusion and)§: } \\
\hline Cardiovascular risk factors $\ddagger$ & $11213(0.71)$ & $237(4.3)$ & 0.60 (0.49 to 0.73$)$ \\
\hline Cerebrovascular disease & $491(0.031)$ & $44(0.55)$ & 0.58 (0.41 to 0.82$)$ \\
\hline
\end{tabular}

$\mathrm{Ml}=$ myocardial infarction; $\mathrm{CAD}=$ coronary artery disease; $\mathrm{PNS}=$ peripheral nervous system; $\mathrm{CHF}=$ congestive heart failure.

*All univariate P values for comparison between those who did and did not have stroke or MI were $<0.001$, except smoking status $(P=0.032)$.

tPer decade.

\#Includes patients who have at least one of $\mathrm{CHF}$, atrial fibrillation, diabetes with chronic complications, or renal failure.

$\S$ To interpret interaction terms, multiply odds ratio for high risk comorbidities or cerebrovascular disease by OR conferred by pRBC transfusion, and then by interaction term OR-for example, patient with cardiovascular risk factors transfused two units would have OR for stroke or MI of

$2.37 \times 2.43 \times 0.60=3.46$, whereas patient without cardiovascular risk factors two units would have $O R$ of 2.37 , and patient with cardiovascular risk factors who was not transfused would have OR of 2.43. Significant interaction term suggests model over or underestimates ORs for patients with more than one factor in model. In this case, model overestimates ORs for patients with transfusion with history of cardiovascular risk factors or cerebrovascular disease.

Propensity matched analysis confirmed an increased risk of stroke or myocardial infarction in patients who underwent transfusion, with a number needed to treat to harm of 267.

Our findings confirm and extend a recent report by Kamel and colleagues, who found an increase in perioperative stroke/myocardial infarction after transfusion of four or more units of packed red blood cells. ${ }^{2}$ The authors hypothesized that the hemodynamic derangements of hemorrhage contributed to the association with transfusion. Over $90 \%$ of patients who underwent transfusion in our dataset, however, received less than four units, and a sensitivity analysis confirmed the association with stroke/ myocardial infarction is seen even in patients who only received one unit. In that group, an increased odds of stroke/myocardial infarction is less likely to reflect hemorrhagic hypoperfusion. Lending additional support to this idea, a recent study of $2.2 \mathrm{mil}-$ lion percutaneous coronary intervention (PCI) 


\begin{tabular}{|c|c|c|c|c|c|}
\hline Subgroup variable & $\begin{array}{l}\text { Colectomy } \\
\text { (partial and total) }\end{array}$ & $\begin{array}{l}\text { Small bowel } \\
\text { resection }\end{array}$ & $\begin{array}{l}\text { Hip/knee } \\
\text { replacement or } \\
\text { revision }\end{array}$ & $\begin{array}{l}\text { Spine, including } \\
\text { fusion and } \\
\text { laminectomy }\end{array}$ & Hysterectomy \\
\hline No (\%) transfused & $1748(4.6)$ & $647(4.0)$ & $15516(3.6)$ & $3903(2.0)$ & $1747(1.6)$ \\
\hline No (\%) with stroke/MI (\%) & $689(1.8)$ & 309 (1.9) & $1447(0.33)$ & $670(0.34)$ & $115(0.10)$ \\
\hline \multicolumn{6}{|c|}{ Odds ratio for stroke/myocardial infarction $(95 \% \mathrm{CI})$} \\
\hline \multicolumn{6}{|c|}{ pRBC use (units) (reference: 0 units): } \\
\hline 1 & 2.36 (1.33 to 4.19) & 2.05 (0.66 to 6.30) & 1.26 (0.78 to 2.03$)$ & $1.43(0.65$ to 3.14$)$ & 5.21 (1.15 to 23.7) \\
\hline
\end{tabular}

procedures without periprocedural bleeding complications also showed an increased odds of stroke and in-hospital myocardial infarction associated with transfusion, with odds ratios of 8.49 (95\% confidence interval 8.21 to 8.78 ) and 2.38 (2.35 to 2.41), respectively, in a propensity weighted analysis adjusted for comorbidities and indication for the procedure. ${ }^{19}$

\section{Clinical implications}

In vitro data and data from animal and human studies show that packed red blood cell transfusion introduces inflexible hyperaggregable red blood cells, activates the thrombogenic potential of vascular endothelium and platelets, and can initially hamper tissue oxygenation, providing biological plausibility for a link between transfusion and perioperative stroke/myocardial infarction. We and others ${ }^{2}{ }^{19}$ have now found that this association can be shown in large retrospective cohorts, though data from randomized controlled trials are lacking. Although transfusion remains the mainstay of treatment for severe anemia, the pendulum of medical

\begin{tabular}{|c|c|c|c|c|}
\hline \multirow[b]{2}{*}{ Variable } & \multirow[b]{2}{*}{ Whole dataset } & \multicolumn{2}{|c|}{$\begin{array}{l}\text { Propensity score } \\
\text { matched }\end{array}$} & \multirow[b]{2}{*}{$P$ value } \\
\hline & & $\begin{array}{l}\text { Not } \\
\text { transfused }\end{array}$ & Transfused & \\
\hline No of patients & 1583819 & 41421 & 41421 & - \\
\hline No (\%) with stroke/MI & 8044 & $336(0.81)$ & $496(1.1)$ & $<0.001^{\star}$ \\
\hline \multicolumn{5}{|c|}{ Adjusted odds ratios for stroke/MIt } \\
\hline \multicolumn{5}{|c|}{ pRBC use (units) (reference: 0 units): } \\
\hline 1 & $2.33(1.90$ to 2.86$)$ & \multicolumn{2}{|c|}{$1.71(1.31$ to 2.24$)$} & \multirow{4}{*}{$<0.001$} \\
\hline 2 & 2.37 (2.00 to 2.81$)$ & \multicolumn{2}{|c|}{$1.73(1.36$ to 2.20$)$} & \\
\hline 3 & $3.13(2.28$ to 4.31$)$ & \multicolumn{2}{|c|}{2.24 (1.56 to 3.22$)$} & \\
\hline$\geq 4$ & $4.87(3.86$ to 6.14$)$ & \multicolumn{2}{|c|}{$3.16(2.36$ to 4.23$)$} & \\
\hline
\end{tabular}

opinion is swinging toward more judicious use, or avoidance altogether, of this potentially harmful therapy. Surgical anemia is particularly amenable to transfusion-sparing strategies and-more specificallypre-emptive strategies intended to decrease perioperative blood loss or treat and correct anemia before elective surgery. Management of Jehovah's Witnesses through major surgery provides an extreme example of blood sparing approaches, ${ }^{20}$ and coordinated strategies to manage perioperative anemia might improve outcomes. ${ }^{21}$ Thus, the use of allogeneic packed red blood cell transfusion is potentially modifiable by preoperative, ${ }^{20}$ intraoperative, ${ }^{2021}$ and postoperative strategies, ${ }^{20-23}$ and our findings support rigorous clinical study of strategies to reduce transfusions in the interest of harm reduction.

\section{Strengths and limitations of this study}

This large population based dataset allows the robust investigation of rare perioperative complications, like perioperative ischemic stroke and myocardial infarction. Because any absolute increase in risk incurred by those who receive a transfusion would be quite small, these conclusions might not have been demonstrable with a smaller cohort. Indeed, the largest published randomized controlled trials of transfusion thresholds in surgical patients have not found a significant increase in rates of perioperative stroke/myocardial infarction. ${ }^{2223}$ Our findings, however, were robust across two independent statistical methods (that is, hierarchical logistic regression and propensity matching) and among many subgroup and sensitivity analyses. Furthermore, although it is not perfectly representative of the US population, the dataset we used includes a diverse variety of hospitals: urban and rural, community focused through tertiary care, and teaching and non-teaching hospitals. It also is not limited by type of health insurance. Accordingly, these findings are likely to be generalizable to a broad perioperative population.

Our study has some limitations that should be considered when our findings are interpreted. First, as this 
is an analysis of administrative data, detailed clinical data were not available. For example, no preoperative (or postoperative) measurements of hemoglobin are recorded in the dataset. Preoperative anemia is an important risk factor for perioperative complications, probably caused by comorbid medical conditions and/ or as a marker of frailty, though recent research has shown that direct effects of preoperative anemia might be weaker than the impact of mediating factors for adverse outcomes, such as transfusion. ${ }^{24}$ Although we used a validated method of identifying some patients with anemia through the use of Elixhauser comorbidity coding from ICD-9 codes, this could undercount patients with pre-existing anemia. Furthermore, the accuracy of ICD-9 codes depends on the accuracy of collection of administrative/billing data, ${ }^{25}$ a limitation common to large database cohort studies such as this one. As part of its business model, however, Premier undertakes a number of coding validation activities to ensure the cross site consistency and accuracy of data collected in Perspective. The rate of stroke/myocardial infarction events we observed $(0.51 \%)$ is quite similar to the $0.44 \%$ event rate observed in the National Surgical Quality Improvement Program clinical dataset, ${ }^{2}$ suggesting that Premier's quality assurance procedures are effective. Though we had no information on transfusion thresholds or institutional policies regarding transfusions, we attempted to account for variation in practices between institutions by using hierarchical modeling. We also could not adjust for the indication for transfusion (for instance, symptomatic anemia $v$ ongoing blood loss) and could not use the timing of the transfusion (for instance, intraoperative $v$ postoperative) as a surrogate as transfusions were not time stamped within this dataset. Because we had no hemodynamic data, there was also no way to ensure that transfusions were not given as a response to hypotension as a result of hemorrhage, which would potentially increase the risk for stroke or myocardial infarction independently of the transfusion itself, although we attempted to minimize this potential confounder by subgroup analyses. Activation of hemostatic mechanisms can occur in proportion to operative trauma and blood loss (and thus in proportion to transfusion), which could confound the relation between stroke/myocardial infarction and transfusion. Finally, we could not ensure that transfusions did not occur after an early (that is, on the day of surgery or the day after) myocardial infarction or stroke; however, a sensitivity analysis that excluded patients who underwent transfusion on the day after surgery yielded similar results.

\section{Future directions}

Because of the unavoidable possibility of residual confounding, retrospective cohort studies, however large, rarely provide definitive answers to clinical questions. There are, however, no published randomized controlled trials of transfusion threshold designed to study rates of stroke/myocardial infarction as a primary outcome. One potential explanation is that, based on the rates of stroke/myocardial infarction in our propensity matched cohort, a trial of transfusion triggers designed with stroke/myocardial infarction as the primary outcome would require a sample size of over 22000 patients to achieve appropriate two sided statistical power. Even meta-analytic methods might not currently be able to assemble a sufficient sample size. A recent meta-analysis of liberal versus restrictive transfusion strategies assembled 31 trials with only 9813 patients. ${ }^{26}$ Future research into this question could therefore focus on large retrospective cohorts with more detailed clinical data (such as hemoglobin values, estimated intraoperative blood loss, more granular detail of perioperative comorbidities, precise timing of transfusion, and outcomes of interest, etc), in an attempt to deal with residual confounding, until more large randomized controlled trials can be conducted.

\section{Conclusions}

In summary, we found that in a large retrospective cohort of patients undergoing a diverse variety of surgeries, perioperative transfusion of a single unit of red blood cells is associated with an increased risk of perioperative ischemic stroke or myocardial infarction, with an adjusted odds ratio of 2.33 (95\% confidence interval 1.90 to 2.86). Our findings were robust to extensive subgroup and sensitivity analyses. Given the relative safety of restrictive transfusion approaches in critical care and perioperative medicine, ${ }^{26}$ the association we have shown between perioperative transfusion and rare but serious adverse outcomes provides additional support for the pursuit of strategies to avoid transfusion.

We thank Judy Maselli for her assistance with data management and Nancy Hills and I Elaine Allen for their assistance with statistical analysis.

Contributors: All authors contributed to study design. ADA obtained the data from Premier, and ELW analyzed the data under the guidance of HK and ADA. All authors had full access to all of the data in the study. All authors contributed to data interpretation, reviewed and approved the final manuscript, and agree to be accountable for all aspects of the work. ADA is guarantor.

Funding: ELW received funding from the UCSF Department of Anesthesia and Perioperative Care to support this work. ADA is supported by grant number K24HL098372 from the National Heart, Lung and Blood Institute of the National Institutes of Health. Statistical consultation was supported by grant number UL1-TR000004 from the National Institutes of Health. The funding sources had no role in the study design, analysis or interpretation of data, writing of the manuscript, or decision to submit the paper for publication.

Competing interests: All authors have completed the ICMJE Uniform Disclosure at http://www.icmje.org/coi_disclosure.pdf (available on request from the corresponding author) and declare: no support from any organization for the submitted work; no financial relationships with any organizations that might have an interest in the submitted work in the previous three years; no other relationships or activities that could appear to have influenced the submitted work. Ethical approval: Not required.

Data sharing: Patient level data were purchased from Premier and are subject to restrictions on use of data, including data sharing. Statistical code is available from the corresponding author.

Transparency: The senior author, ADA, affirms that the manuscript is an honest, accurate, and transparent account of the study being reported; that no important aspects of the study have been omitted; and that any discrepancies from the study as planned (and, if relevant, registered) have been explained. 
This is an Open Access article distributed in accordance with the Creative Commons Attribution Non Commercial (CC BY-NC 4.0) license, which permits others to distribute, remix, adapt, build upon this work non-commercially, and license their derivative works on different terms, provided the original work is properly cited and the use is non-commercial. See: http://creativecommons.org/licenses/ by-nc/4.0/.

1 Whitaker B. The 2011 national blood collection and utilization survey report. Department of Health and Human Services, 2011.

2 Kamel H, Johnston SC, Kirkham JC, et al. Association between major perioperative hemorrhage and stroke or Q-wave myocardial infarction. Circulation 2012;126:207-12.

3 Sharifpour M, Moore LE, Shanks AM, Didier TJ, Kheterpal S, Mashour GA. Incidence, predictors, and outcomes of perioperative stroke in noncarotid major vascular surgery. Anesth Analg 2013;116:424-34

4 Musallam KM, Rosendaal FR, Zaatari G, et al. Smoking and the risk of mortality and vascular and respiratory events in patients undergoing major surgery. JAMA Surg 2013;148:755-62.

5 Devereaux PJ, Xavier D, Pogue J, et al. Characteristics and short-term prognosis of perioperative myocardial infarction in patients undergoing noncardiac surgery: a cohort study. Ann Intern Med 2011;154:523-8

6 Relevy H, Koshkaryev A, Manny N, Yedgar S, Barshtein G. Blood banking-induced alteration of red blood cell flow properties. Transfusion 2008;48:136-46

7 Bennett-Guerrero E, Veldman TH, Doctor A, Telen MJ, Ortel TL, Reid TS, et al. Evolution of adverse changes in stored RBCs. Proc Natl Acad Sci USA 2007;104:17063-8.

8 Silvain J, Abtan J, Kerneis M, et al. Impact of red blood cell transfusion on platelet aggregation and inflammatory response in anemic coronary and noncoronary patients: the TRANSFUSION-2 study (impact of transfusion of red blood cell on platelet activation and aggregation studied with flow cytometry use and light transmission aggregometry). J Am Coll Cardiol 2014:63:1289-96.

9 Gramm J, Smith S, Gamelli RL, Dries DJ. Effect of transfusion on oxygen transport in critically ill patients. Shock 1996;5:190-3.

10 Marik PE, Sibbald WJ. Effect of stored-blood transfusion on oxygen delivery in patients with sepsis. JAMA 1993;269:3024-9.

11 Tsai AG, Cabrales P, Intaglietta M. Microvascular perfusion upon exchange transfusion with stored red blood cells in normovolemic anemic conditions. Transfusion 2004:44:1626-34

12 Elixhauser A, Steiner C, Harris DR, Coffey RM. Comorbidity measures for use with administrative data. Med Care 1998;36:8-27.

13 Myerburg RJ, Junttila MJ. Sudden cardiac death caused by coronary heart disease. Circulation 2012;125:1043-52.
14 Lee S, Han I, Kahng J, Kim Y, Shin DS, Han EK. Organization of maximum surgical blood order schedule (MSBOS) according to the international classification of diseases, ninth revision, clinical modification (ICD-9-CM). Korean J Blood Transfus 2008;19:15-24

15 Maximum surgical blood ordering schedule [MSBOS]-PICIS. Transfusion Medicine and Blood Banking Service, University of California, 24 February 2014. http://labmed.ucsf.edu/labmanual/ clinlab/dnld/MSBOS.pdf

16 Greenland S, Drescher K. Maximum likelihood estimation of the attributable fraction from logistic models. Biometrics 1993:49:865-72.

17 Lin DY, Psaty BM, Kronmal RA. Assessing the sensitivity of regression results to unmeasured confounders in observational studies. Biometrics 1998:54:948-63.

18 Sturmer T, Schneeweiss S, Avorn J, Glynn RJ. Adjusting effect estimates for unmeasured confounding with validation data using propensity score calibration. Am J Epidemiol 2005;162:279-89.

19 Sherwood MW, Wang Y, Curtis JP, Peterson ED, Rao SV. Patterns and outcomes of red blood cell transfusion in patients undergoing percutaneous coronary intervention. JAMA 2014;311:836-43.

20 McCartney S, Guinn N, Roberson R, Broomer B, White W, Hill S. Jehovah's witnesses and cardiac surgery: a single institution's experience. Transfusion 2014;54:2745-52

21 LaPar DJ, Crosby IK, Ailawadi G, et al. Blood product conservation is associated with improved outcomes and reduced costs after cardiac surgery. J Thorac Cardiovasc Surg 2013;145:796-804.

22 Hajjar LA, Vincent JL, Galas FR, et al. Transfusion requirements after cardiac surgery: the TRACS randomized controlled trial. JAMA 2010;304:1559-67.

23 Carson JL, Terrin ML, Noveck H, et al. Liberal or restrictive transfusion in high-risk patients after hip surgery. N Engl I Med 2011;365:2453-62.

24 Saager L, Turan A, Reynolds LF, Dalton JE, Mascha EJ, Kurz A. The association between preoperative anemia and 30-day mortality and morbidity in noncardiac surgical patients. Anesth Analg 2013;117:909-15.

25 O'Malley KJ, Cook KF, Price MD, Wildes KR, Hurdle JF, Ashton CM. Measuring diagnoses: ICD code accuracy. Health Serv Res 2005; $40: 1620-39$

26 Holst LB, Petersen MW, Haase N, Perner A, Wetterslev J. Restrictive versus liberal transfusion strategy for red blood cell transfusion: systematic review of randomised trials with meta-analysis and trial sequential analysis. BMJ 2015;350:h1354

(C) BMJ Publishing Group Ltd 2015

Appendix: Supplementary tables A and B 